\title{
Restoration of renal function in zebrafish models of ciliopathies
}

\author{
Jonathan L. Tobin • Philip L. Beales
}

Received: 21 February 2008 /Revised: 23 April 2008 / Accepted: 16 May 2008 / Published online: 5 July 2008

(C) The Author(s) 2008

\begin{abstract}
The ciliopathies are a class of rare human genetic disease whose aetioligies lie in defective primary cilia. Typical ciliopathies include Bardet-Biedl syndrome (BBS), nephronophthisis (NPHP), Jeune, Joubert, oro-facial-digital (OFD1) and Meckel (MKS) syndromes. All ciliopathies have the common denominator of renal disease, often including tubular cysts. In this study, we have modelled a range of ciliopathies in zebrafish and shown in all cases that knocking down these genes causes cystic lesions in the kidney. We have identified two drugs, rapamycin and roscovitine, which ameliorate the renal phenotype, both morphologically and functionally. This is the first study in which zebrafish has been used to identify potential therapeutic modalities for ciliopathic renal disease, and the results pave the way for further investigations in mammalian models.
\end{abstract}

Keywords Ciliopathy · Kidney cysts · Rapamycin ·

Roscovitine $\cdot$ Therapeutics

\section{Introduction}

Primary cilia function to detect signalling molecules and mechanical stimuli, such as fluid flow. Mutations in genes encoding some of the approximately 1000 ciliary proteins cause ciliopathies. Renal disease is a common denominator of ciliopathies, often presenting with tubular cysts, lobulation and clubbing $[1,2]$. Published mouse models exist for

J. L. Tobin · P. L. Beales $(\square)$

Molecular Medicine Unit, UCL Institute of Child Health,

30 Guilford Street,

London WC1N 1EH, UK

e-mail: p.beales@ucl.ac.uk several ciliopathy genes (Bbs1, 2, 4, 6; Ofdl; Alms 1, Pkhd), and they develop renal cysts but often not until several months of age [3].

The zebrafish is developing as a valid model for investigating human cystic kidney diseases [4]. The zebrafish pronephros develops after 2 days post-fertilisation (dpf), and the presence of cysts is easily screened at $3 \mathrm{dpf}$. These cysts often compromise renal function, resulting in body oedema by $4 \mathrm{dpf}$. Transparency of the embryos makes morphological scoring rapid, and the large clutches enables scalability. Ex vivo development allows therapeutic compounds to be added directly to the water in which the eggs are incubated [5]. Our aim is to create fish models for most of the known human ciliopathies, screen them for a kidney phenotype and then try to identify compounds that could prevent cyst formation and potentially restore function.

\section{Methods}

Between 2 and $4.5 \mathrm{ng}$ of translation and splice-blocking morpholino oligonucleotides (MOs) (Gene Tools, Philomath, OR) were used against the following genes (disease caused by human mutations in brackets): bbs $4, b b s 6, b b s 8$ (BardetBiedl syndrome); nphp2 (nephronophthisis); nphp5 (SeniorLoken syndrome); nphp6 (Joubert syndrome); mks1, mks3 (Meckel syndrome); ofd1 (oro-facial-digital syndrome type I). The MO sequences are available on request. Renal lesion sizes were quantified by drawing an outline on a lateral image of the swelling at $5 \mathrm{dpf}$ using AXIOIMAGER ver. 4.4 software (Carl Zeiss Microimaging, Thornwood, NY).

Rapamycin (2-100 nM; Sigma, St. Louis, MO) and roscovitine (5-100 $\mu M$; Sigma) were dissolved in $1 \%$ DMSO and added directly to fish water at $24 \mathrm{hpf}$. Controls were treated with DMSO only. For the functional assay, 
embryos were injected with MO or control MO and, $24 \mathrm{~h}$ later, treated with drug or vehicle. At $72 \mathrm{hpf}$, they were anaesthetised in tricaine, immobilised in $2.5 \%$ methyl cellulose, and $1 \mathrm{nl}$ of $10-\mathrm{kDa}$ rhodamine-dextran (Invitrogen, Carlsbad, CA) was injected into the pericardium. Images of the fluorescent embryos were collected at 1,3 , and $24 \mathrm{~h}$ post-injection. A region of $100 \times 100$ pixels containing the heart was selected, and the fluorescence intensity measured using IMAGEJ software (available at: http://rsb.info.nih.gov/ij). At least ten embryos were measured for each treatment, and average fluorescent intensity at each timepoint was measured. The percentage of fluorescence remaining after $24 \mathrm{hpf}$ was used to determine the efficiency of renal filtration.

\section{Results}

We recently showed that mutations in a core intraflagellar transport (IFT) gene, IFT80, cause Jeune syndrome, a ciliopathy featuring skeletal dysplasia, renal cysts, polydactyly and situs inversus [6]. The zebrafish morphant showed cystic kidneys, random body situs (our unpublished data) and a down-turned body-axis; all features reminiscent of bona fide ciliary mutants. Other previously published zebrafish ciliopathy morphants, including $b b s 4, b b s 6$ and nphp2, also show similar phenotypes [7,8].

Given the recapitulation of kidney anomalies in the ift80 fish model, we extended our study to include other representative ciliopathy candidates, such as Bardet-Biedl syndrome genes $b b s 4, b b s 6$ and $b b s \&$; Meckel syndrome genes $m k s 1$ and $m k s 3$; oro-facial-digital gene ofdl and the nephronophthisis genes $n p h p 2, n p h p 5$ and nphp6. Injection of all these MOs into zebrafish produced a visible cyst in the kidney by $3 \mathrm{dpf}$ (see [6] for ift80; data not shown for others) and severe oedema by $5 \mathrm{dpf}$ (Fig. 1a-h). The efficiency of knockdown was validated by reverse transcriptase (RT)-PCR (not shown), and specificity proven by phenotypic rescue by coinjection with mouse Ift80 mRNA (see [3]).

Several drugs have been shown to retard or reverse the growth of kidney cysts, but none have ever been tested in trials for the treatment of ciliopathy-associated renal disease. The anti-proliferative rapamycin is a small molecule inhibitor of the mTOR pathway [9]. mTOR interacts with the C-terminus of polycystin-1 (PC1), a protein mutated in autosomal dominant polycystic kidney disease which localises to the cilium [10]. We reasoned that rapamycin might be a good candidate to test on our ciliopathy models and hypothesised that dysfunction of ciliopathy proteins could result in the mislocalisation of $\mathrm{PC} 1$ and consequent inappropriate activation of mTOR in the kidney. If this were the case, then rapamycin would ameliorate cyst formation in our morphants.
To test this, we added $10 \mathrm{n} M$ rapamycin to the zebrafish water at $24 \mathrm{hpf}$. The addition of rapamycin to uninjected controls had no effect (Fig. 1a,a'). The left-hand column of Fig. 1 (Fig. 1a-g) shows renal oedema at $5 \mathrm{dpf}$ in every ciliopathy morphant tested that had been treated with vehicle (DMSO) alone. The right-hand column (Fig. 1 $1 a^{\prime}-g^{\prime}$ ) shows how every morphant treated with rapamycin shows almost complete restoration of renal size and morphology. This is quantified in Fig. 1i where the size of the oedematous swelling is measured with and without rapamycin for all morphants tested. The other phenotypes caused by the MO (curly tail, cardiac oedema, otolith defects) are unaffected by the addition of rapamycin, suggesting a kidney-specific effect.

In the absence of rapamycin, $100 \%$ of $m k s 1$ morphants, the mutations of which are lethal in humans, died by $5 \mathrm{dpf}$ and necrosed (Fig. 1f). However, upon the addition of rapamycin, $75 \%$ of $m k s 1$ morphants survived (Fig. 1f'). This suggests that rapamycin was able to prevent the early lethality of $m k s 1$ morphants.

We extended the study to include roscovitine, a cyclindependent kinase inhibitor recently shown to inhibit cyst progression in murine models of polycystic kidney disease [11]. The addition of roscovitine at $6 \mathrm{hpf}$ caused embryos to die before the tail-bud stage due to widespread apoptosis (data not shown). When roscovitine was added at $24 \mathrm{hpf}$, we observed partial amelioration of the renal phenotype and cardiac oedema (Fig. 1h). Figure $1 \mathrm{j}$ shows that roscovitine reduced the size of swelling in the kidney in a dosedependent fashion, but the effect is far less dramatic than with rapamycin.

It is clear that roscovitine and rapamycin were able to rescue the oedema caused by the cysts. That rapamycin rescued the lethality of the $m k s 1$ morphants suggests that it also has some positive effect on renal function. To assay the effect on renal function, we adapted the protocol of Hentschel et al. [12]. We injected the hearts of control and morphant embryos, both treated and untreated with drugs, with rhodamine-labelled $10-\mathrm{kDa}$ dextran and followed the passage of fluorescent dextran out of the embryo. If kidney function is normal, the dextran will be filtered through the glomerulus and excreted in the urine, resulting in a disappearance of fluorescence $24 \mathrm{~h}$ after injection. This was apparent in control embryos (Fig. $1 \mathrm{k}, \mathrm{k}^{\prime}$ and $\mathrm{m}, \mathrm{m}^{\prime}$ ), but in ift 80 morphants only around $50 \%$ of the dextran was excreted from the embryo, indicating a $50 \%$ loss of filtration capacity (Fig. 11,1' and n, n').

An accumulation of dextran in the pronephric duct implies a backing-up in the pronephros, likely due to a reduced ability to filter the bloodstream (Fig. 1n'). This impairment of filtration is unlikely to be a secondary effect of compromised circulation as the dextran had diffused into the peripheral vasculature of the morphant embryos just as 

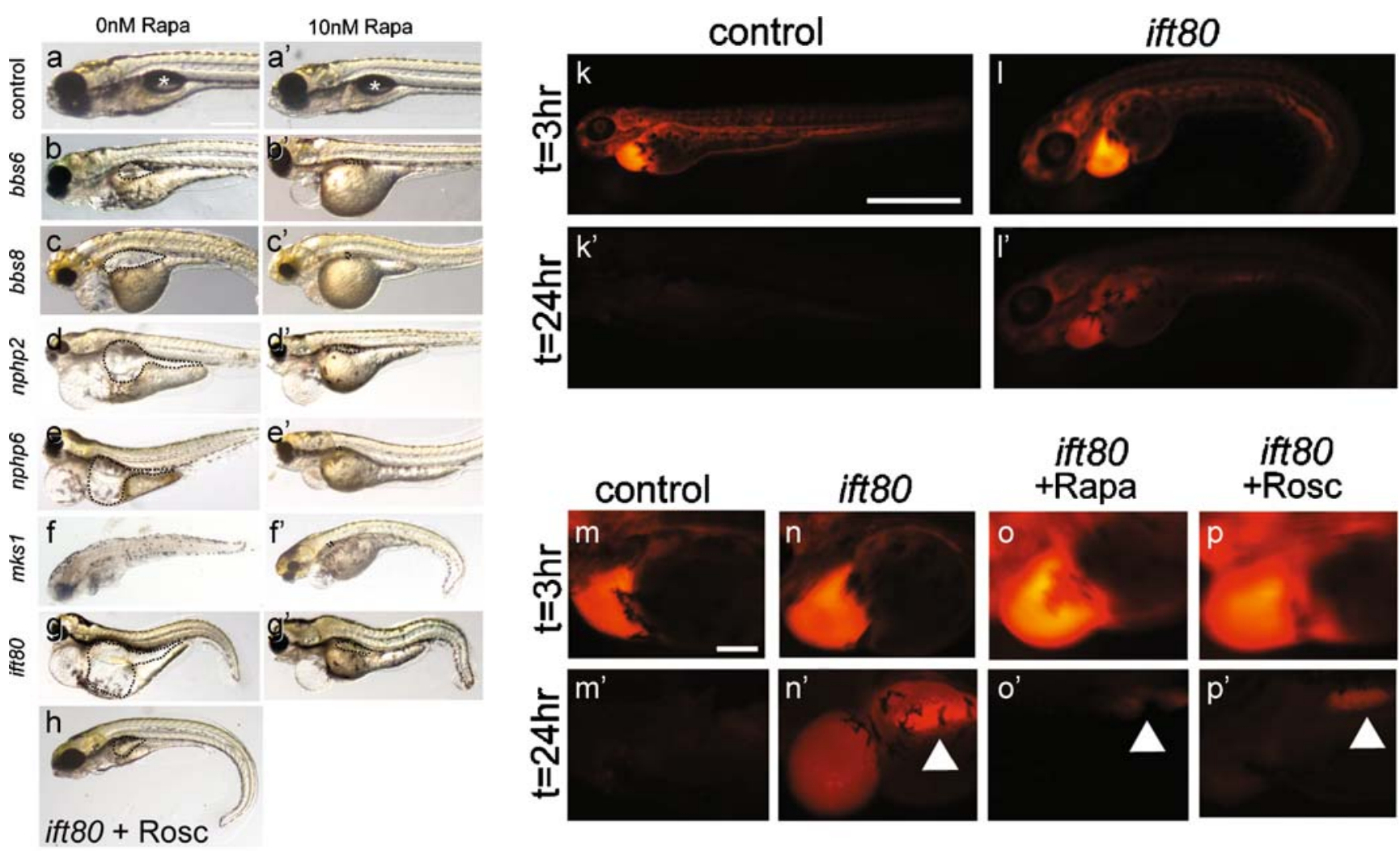
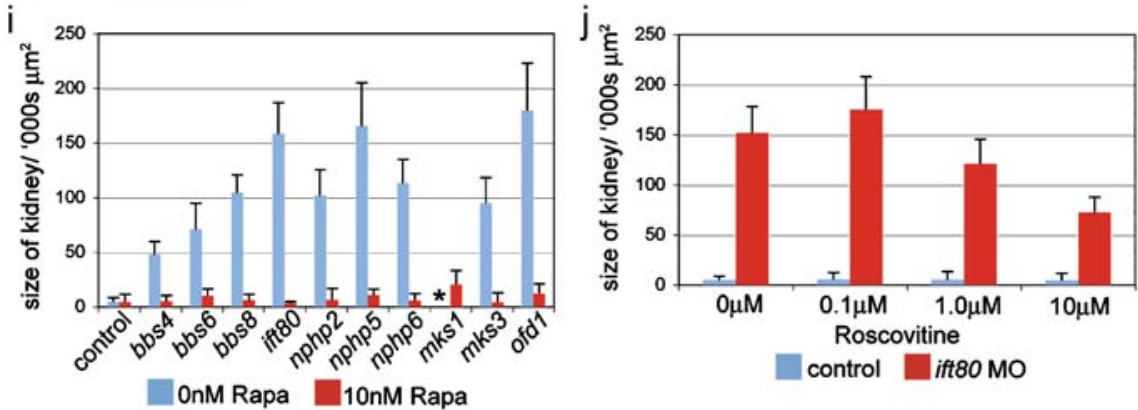

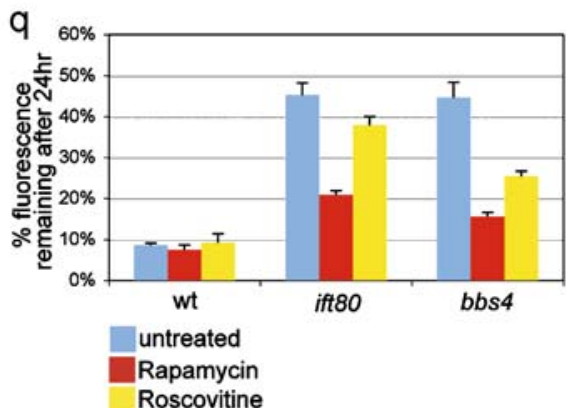

Fig. 1 a, a' Addition of $10 \mathrm{n} M$ rapamycin (Rapa) to control zebrafish has no effect on morphology at $5 \mathrm{dpf}$. Asterisk indicates the swim bladder (not visible in morphant embryos). b-e' bbs6, bbs $8, n p h p 2$ and nphp6 all show a dramatic reduction in kidney oedema (outlined) following the addition of Rapa. Other morphological phenotypes are unaffected by the treatment. f, $\mathbf{f}^{\prime} m k s 1$ morphants all die by $5 \mathrm{dpf}$ without Rapa, but $75 \%$ survive and show reduced kidney swelling after the addition of Rapa. $\mathbf{g}, \mathbf{g}^{\prime}$ ift 80 morphant kidney phenotype is also reversed by Rapa. Note that the pericardial oedema and curved body axis are unaffected by the drug. $\mathbf{h}$ Roscovitine (Rosc) also reduces the size of the kidney swelling but to a much lesser extent than Rapa. It also appears to ameliorate some of the cardiac oedema. i Graph showing the lateral surface area of the kidney oedema with and without Rapa for all ten genes knocked down. In all cases, the addition of Rapa reduced the kidney to levels similar to that of the controls. $\mathbf{j}$ Addition of a range of Rosc concentrations to ift 80 morphants shows dosage-dependency, but even at high doses it is unable to reduce kidney swelling to the extent of Rapa ( $M O$ morpholino oligonucleotide). $\mathbf{k}$ Fluorescent image of an embryo $3 \mathrm{~h}$ after injection of rhodamine dextran into the heart. Fluorescence disperses throughout the circulation. $\mathbf{k}^{\prime}$ The same embryo
$24 \mathrm{~h}$ after injection shows the complete absence of fluorescence as it has all been evacuated by the kidney. I Fluorescent dextran perfuses systemically throughout the ift 80 morphant to the same extent as controls $3 \mathrm{~h}$ after injection. $\mathbf{l}^{\prime}$ The same embryo $24 \mathrm{~h}$ after injection still has residual fluorescence in the circulation. $\mathbf{m}, \mathbf{m}^{\prime}$ Close-up of the heart of a control zebrafish showing the elimination of fluorescence after $24 \mathrm{~h}$. n, $\mathbf{n}^{\prime}$ Persistence of fluorescence after $24 \mathrm{~h}$ in ift 80 morphants, particularly in the pronephros (arrowhead). $\mathbf{0}, \mathbf{o}^{\prime}$ treatment with Rapa reduces the residual fluorescence in morphants, particularly in the heart, but some still persists in the pronephros (arrowhead). p, $\mathbf{p}^{\prime}$ treatment with Rosc also partially rescues the filtering defect but less so than Rapa (arrowhead). q Graph quantifying the percentage of original fluorescence remaining in the kidney $24 \mathrm{~h}$ after injection. Addition of drugs has no effect on control embryos. Fluorescence is reduced to a similar level as that in untreated ift 80 and $b b s 4$ morphants, but both drugs have a slightly more marked effect on bbs 4 than ift 80 morphants. This graph also shows that Rapa is more effective than Rosc in this system. In all graphs, error bars represent the standard error of the mean $(n \geq 10$ embryos in all cases). Bars (a-h) $1 \mathrm{~mm},\left(\mathbf{k}-\mathbf{I}^{\prime}\right) 1 \mathrm{~mm},\left(\mathbf{m}-\mathbf{p}^{\prime}\right) 100 \mu \mathrm{m}$ 
quickly as in the controls (Fig. 11). Despite cardiac oedema in the morphants, the heart beat at the same rate and with the contractile force as in the controls (data not shown).

Treatment with rapamycin was able to partially rescue the filtering capacity of the kidney, as evidenced by a faint fluorescence remaining in the heart and pronephros $24 \mathrm{~h}$ post-injection (Fig. 1o, ' '). When roscovitine was added to these morphants, there was also a significant reduction in retained dextran compared to untreated fish, but there was more residual fluorescence in the heart and pronephros than in rapamycin-treated embryos (Fig. 1p, $\mathrm{p}^{\prime}$ ). Therefore, roscovitine is not as effective at restoring renal function in the morphant as rapamycin.

These functional assays were repeated using bbs 4 morphants (and $n p h p 2$, not shown), as another example of a ciliopathy gene causing a different disease when mutated in humans. Figure 1q shows the amount of fluorescence remaining in the heart $24 \mathrm{~h}$ post-injection: $9 \%$ of the dextran remains in the controls, but in both ift 80 and bbs 4 morphants, only about $55 \%$ is removed. Rapamycin had no effect on kidney function in the controls, but it did improve filtering capacity by approximately 2.3 -fold and approximately threefold in ift 80 and bbs 4 morphants, respectively, as calculated by comparing the amount of fluorescence remaining in the heart with or without drug treatment.

The addition of roscovitine improved filtering capacity by approximately 1.2 -fold and approximately 1.8 -fold in ift 80 and bbs 4 morphants, respectively. These data suggest that the bbs 4 morphant kidney phenotype is less severe than the ift 80 one as the function is restored to a greater extent. It also suggests that rapamycin is twice as effective at restoring renal function in zebrafish as roscovitine. Rapamycin had a greater effect on functional restoration when added at $24 \mathrm{hpf}$ than at $4 \mathrm{hpf}$.

\section{Discussion}

The studies reported here validate the zebrafish as a model for human ciliopathies. As there is currently a shortage of mouse models for ciliopathies, many of which suffer from embryonic lethality, have variable temporal cyst development and, moreover, must often be euthanised and sectioned to reveal the cysts, it is far more efficient to screen the phenotypes in the fish [13]. We have demonstrated the virtue of the fish as a preclinical drug screening tool capable of quantitatively differentiating between the efficacy of two different compounds, with the potential to assay functional repair as well as morphological rescue. We have also observed similar levels of renal structural and functional repair with another known anti-cyst drug, paclitaxel (data not shown).

Renal disease is the greatest contributor to morbidity and mortality in ciliopathy patients [2]. However, as the ciliopathies are rare diseases with relatively few patients, there is little commercial incentive to develop orphan drugs and perform clinical trials. Nevertheless, it is important to develop therapies to treat these patients. This preliminary study provides proof of principle for a high-throughput screen using a small-molecule library to identify more compounds. These can then be tested on mouse models with the ultimate aim of treating ciliopathy patients. The approach also validates the use of pre-existing therapies for new diseases that may have similar pathomechanisms to polycystic kidney disease.

Acknowledgements Thanks to Masazumi Tada for provision of zebrafish injection facilities and to Adrian Woolf for helpful discussions. This work was funded by the Medical Research Council (JLT) and the Wellcome Trust (PLB).

Open Access This article is distributed under the terms of the Creative Commons Attribution Noncommercial License which permits any noncommercial use, distribution, and reproduction in any medium, provided the original author(s) and source are credited.

\section{References}

1. Badano JL, Mitsuma N, Beales PL, Katsanis N (2006) The ciliopathies: an emerging class of human genetic disorders. Annu Rev Genomics Hum Genet 7:125-148

2. Tobin JL, Beales PL (2007) Bardet-Biedl syndrome: beyond the cilium. Pediatr Nephrol 22:926-936

3. Beales PL (2005) Lifting the lid on Pandora's box: the BardetBiedl syndrome. Curr Opin Genet Dev 15:315-323

4. Drummond IA (2005) Kidney development and disease in the zebrafish. J Am Soc Nephrol 16:299-304

5. Zon LI, Peterson RT (2005) In vivo drug discovery in the zebrafish. Nat Rev Drug Discov 4:35-44

6. Beales PL, Bland E, Tobin JL, Bacchelli C, Tuysuz B, Hill J, Rix S, Pearson CG, Kai M, Hartley J, Johnson C, Irving M, Elcioglu N, Winey M, Tada M, Scambler PJ (2007) IFT80, which encodes a conserved intraflagellar transport protein, is mutated in Jeune asphyxiating thoracic dystrophy. Nat Genet 39:727-729

7. Gerdes JM, Liu Y, Zaghloul NA, Leitch CC, Lawson SS, Kato M, Beachy PA, Beales PL, DeMartino GN, Fisher S, Badano JL, Katsanis N (2007) Disruption of the basal body compromises proteasomal function and perturbs intracellular Wnt response. Nat Genet 39:1350-1360

8. Otto EA, Schermer B, Obara T, O'Toole JF, Hiller KS, Mueller AM, Ruf RG, Hoefele J, Beekmann F, Landau D, Foreman JW, Goodship JA, Strachan T, Kispert A, Wolf MT, Gagnadoux MF, Nivet $H$, Antignac C, Walz G, Drummond IA, Benzing T, Hildebrandt F (2003) Mutations in INVS encoding inversin cause nephronophthisis type 2, linking renal cystic disease to the function of primary cilia and left-right axis determination. Nat Genet 34:413-420

9. Tao Y, Kim J, Schrier RW, Edelstein CL (2005) Rapamycin markedly slows disease progression in a rat model of polycystic kidney disease. J Am Soc Nephrol 16:46-51

10. Shillingford JM, Murcia NS, Larson CH, Low SH, Hedgepeth R, Brown N, Flask CA, Novick AC, Goldfarb DA, Kramer-Zucker A, Walz G, Piontek KB, Germino GG, Weimbs T (2006) The mTOR 
pathway is regulated by polycystin-1, and its inhibition reverses renal cystogenesis in polycystic kidney disease. Proc Natl Acad Sci USA 103:5466-5471

11. Bukanov NO, Smith LA, Klinger KW, Ledbetter SR, IbraghimovBeskrovnaya O (2006) Long-lasting arrest of murine polycystic kidney disease with CDK inhibitor roscovitine. Nature 444:949-952
12. Hentschel DM, Park KM, Cilenti L, Zervos AS, Drummond I, Bonventre JV (2005) Acute renal failure in zebrafish: a novel system to study a complex disease. Am J Physiol Renal Physiol 288:F923-F929

13. Hentschel DM, Bonventre JV (2005) Novel non-rodent models of kidney disease. Curr Mol Med 5:537-546 